\title{
灯盏花乙素半合成柳穿鱼叶苷研究
}

\author{
颜世强谢明现王玉杰李英霞* \\ (复旦大学药学院 上海 201203)
}

\begin{abstract}
摘要 柳穿鱼黄素及柳穿鱼叶苷作为中药大蓟中黄酮及黄酮甘类的代表性化合物, 具有非常重要的生物活性. 以商业 易得的灯戞花乙素为原料，经羧基酯化、酚羟基选择性甲基化及糖苷键水解 3 步反应，以 $69.5 \%$ 的总收率制备得到柳穿 鱼黄素. 随后，以得到的柳穿鱼黄素为糖基受体、全苯甲酰基保护的澳代芸香糖为糖基供体完成了柳穿鱼叶苷的化学 合成.

关键词 灯戞花乙素; 柳穿鱼黄素; 柳穿鱼叶苷; 黄酮类化合物; 半合成
\end{abstract}

\section{Research on the Semi-synthesis of Pectolinarin from Scutellarin}

\author{
Yan, Shiqiang Xie, Mingxian Wang, Yujie Li, Yingxia* \\ (School of Pharmacy, Fudan University, Shanghai 201203)
}

\begin{abstract}
As the representative flavonoid and flavonoid glycoside of Chinese medicine Cirsium japonicum, pectolinarigenin and pectolinarin have important bioactivities. In this paper, the efficient semi-synthesis of pectolinarigenin had been achieved starting from commercially available scutellarin via a linear reaction sequence of 3 steps with the overall yield of $69.5 \%$, wherein carboxyl esterification, selective hydroxy protecting, and glycosidic bond hydrolyzation were used. Afterwards, the chemical synthesis of pectolinarin had been accomplished by the glycosylation of pectolinarigenin and benzobromorutinose.

Keywords scutellarin; pectolinarigenin; pectolinarin; flavonoids; semi-synthesis
\end{abstract}

黄酮类化合物是植物在长期自然选择过程中产生 的一类次生代谢产物 ${ }^{[1]}$. 黄酮类化合物作为重要的天然 多酚类产物, 不仅广泛存在于高等植物，也存在于许多 低等植物中, 尤其在芸香科、豆科、石南科、唇形科等 高等绿色植物中的分布较多 ${ }^{[2]}$. 黄酮类化合物不仅数量 繁多, 而且结构类型复杂多样. 黄酮类化合物结构类型 的复杂多样性注定了其具有广谱的药理活性, 例如抗氧 化、抗肿瘤、抗炎、抗病毒、免疫调节、解毒护肝和细 胞保护等 ${ }^{[3 \sim 0]}$. 同时黄酮类化合物因其相对较低的毒性, 在临床应用上对人类的肿瘤、抗衰老、心血管疾病以及 退行性疾病的预防和治疗发挥了重要作用. 随着越来越 多的黄酮类化合物及其药理功能的发现, 黄酮类化合物 已成为国内外天然药物研究、开发和利用的热点 ${ }^{[11,12]}$.

中药大蓟为菊科植物蓟 (Cirsium japonicum DC.) 的 干燥地上部分. 大蓟性甘、苦、凉, 具有凉血止血、行 瘀消肿、降血压、抗菌等药理作用, 是一种应用范围广, 临床疗效显著的止血药. 大蓟的化学成分复杂, 到目前
为止, 已从大蓟中分离得到 100 多种化合物, 主要有黄 酮及黄酮苷类、挥发油类、长链炔醇类、木脂素类及其 他类化合物. 其中黄酮和黄酮苷类化合物是大蓟所含成 分中最多的一类，也是目前发现的主要活性成分. 在大 蓟所含有的黄酮和黄酮苷类化合物中，柳穿鱼黄素及柳 穿鱼叶苷是其代表性化合物，同时也是大藓中主要的止 血成分 ${ }^{[13]}$. 现有研究表明: 柳穿鱼黄素具有止血、抗

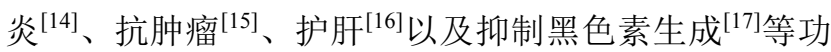
效. 同样作为柳穿鱼黄素芸香糖苷的柳穿鱼叶苷亦具有 抗氧化 ${ }^{[18]}$ 、抗炎 ${ }^{[14 b]}$ 、抗肿瘤 ${ }^{[15,19]}$ 、降糖 ${ }^{[18]} 、$ 护肝 ${ }^{[16]}$ 以 及抑制黑色素生成 ${ }^{[17]}$ 等生物活性.

在天然产物的生物资源合理利用过程中，由含量较 高、提取分离较为容易的天然产物半合成生物活性更强 的类似物与衍生物甚至已上市药物，具有来源丰富、合 成步骤少、生产成本低等优点. 对现代药物的研究与开 发具有重要意义 ${ }^{[20,21]}$.

柳穿鱼黄素的全合成已有文献报道 ${ }^{[22]}$, 采用全合

* Corresponding author. E-mail: liyx417@fudan.edu.cn

Received July 27, 2018; revised September 14, 2018; published online October 14, 2018.

Project supported by the National Natural Science Foundation of China (No. 81773576).

国家自然科学基金(No. 81773576)资助项目. 
成的方法来制备柳穿鱼黄素存在反应步骤较多, 收率较 低等不足. 本文以商业可得的灯盏花乙素为原料半合成 了柳穿鱼黄素及柳穿鱼叶苷, 为它们进一步的生物活性 研究奠定物质基础.<smiles>COc1ccc(-c2cc(=O)c3c(O)c(OC)c(O)cc3o2)cc1</smiles>

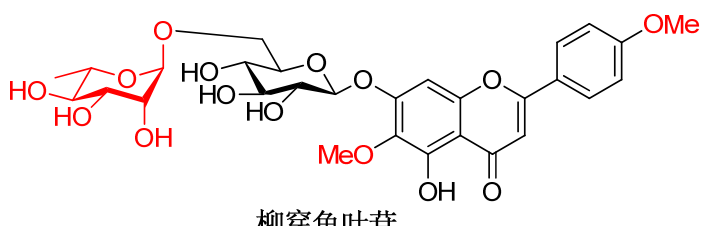

柳穿鱼叶苷

图 1 灯盏花乙素、柳穿鱼黄素及柳穿鱼叶苷的结构

Figure 1 Structure of scutellarin, pectolinarigenin and pectolinarin

\section{1 结果与讨论}

柳穿鱼黄素及柳穿鱼叶苷的逆合成分析(图 2)如下: 柳穿鱼叶苷的制备可通过柳穿鱼黄素 5 与芸香糖糖基供 体 10 在相转移催化下的糖苷化后脱除糖环上的保护基
实现. 柳穿鱼黄素 5 可通过商业易得的灯盏花乙素 $\mathbf{2}$ 经 羧基酯化、酚羟基选择性甲基化以及糖苷水解 3 步反应 制备得到; 芸香糖糖基供体 10 则经 $L$-鼠李糖糖基供体 12 与葡萄糖糖基受体 $\mathbf{8}$ 在酸性催化下的糖苷化后端基 溴代得到.

基于上述反合成分析，柳穿鱼黄素 $\mathbf{5}$ 的合成路线如 Scheme 1 所示. 以商业易得的灯盏花乙素 2 为起始原料, 首先在 10 equiv.氯化亚砜催化下将葡萄糖醛酸的羧基酯 化，以 $93 \%$ 的收率得到灯或花乙素甲酯 $\mathbf{3}^{[23]}$; 化合物 $\mathbf{3}$ 中含有 3 个酚羟基以及 3 个醇羟基，利用酚羟基与醇羟 基之间反应活性的差异，可实现两者之间的选择性甲基 化. 黄酮环上的 5-位酚羟基由于分子内氢键的存在, 致 使其反应活性相对较弱，故以 $N, N$-二甲基甲酰胺(DMF) 为溶剂、无水碳酸钾为缚酸剂, 控制碘甲烷的加入量, 可以对黄酮环上的 6,4'-位酚羟基选择性甲基化保护，使 用 $95 \%$ 乙醇重结晶，以 $84 \%$ 的收率得到淡黄色固体化合 物 4 ; 在此需要指出的是, 我们曾尝试碱性条件下以灯 戛花乙素 2 为原料一步反应制备得到化合物 4 , 遗憾的 是反应效果较差, 很难得到我们需要的目标产物. 室温 下将灯戞花乙素溶于 DMF，在 4 equiv.无水碳酸钾的存 在下, 缓慢向反应体系中滴加 3.5 equiv. 的碘甲烷, 滴加 完毕反应体系很快成为果冻状，致使反应难以后继发 生. 化合物 4 在酸性条件下糖苷键水解, 以 $89 \%$ 的收率

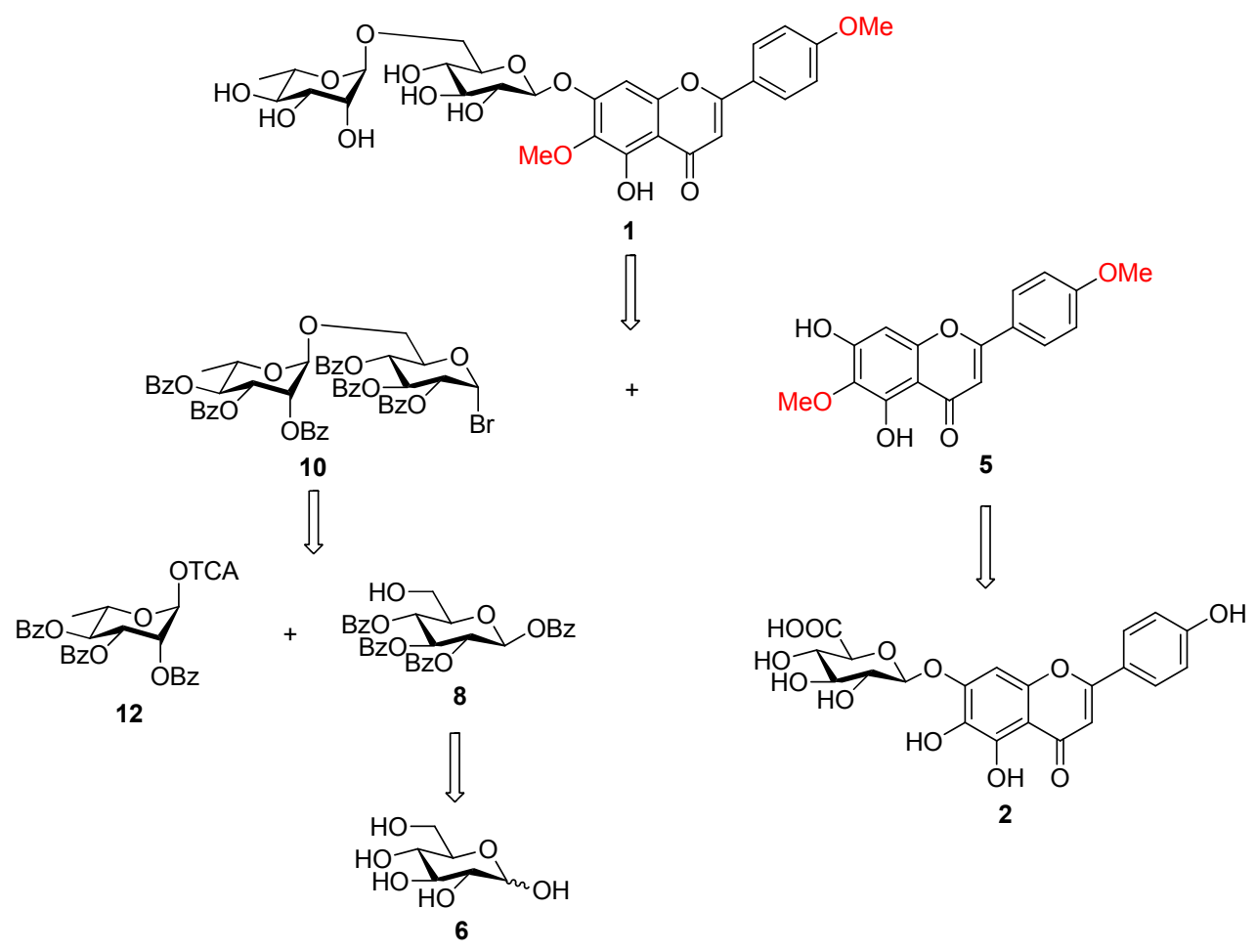

图 2 柳穿鱼黄素及柳穿鱼叶苷的逆合成分析

Figure 2 Retrosynthetic analysis of pectolinarigenin and pectolinarin 


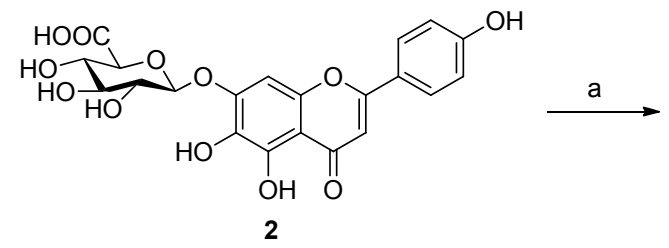<smiles>COC(=O)C1(O)OC(Oc2cc3oc(-c4ccc(O)cc4)cc(=O)c3c(O)c2O)C(O)C1C</smiles>

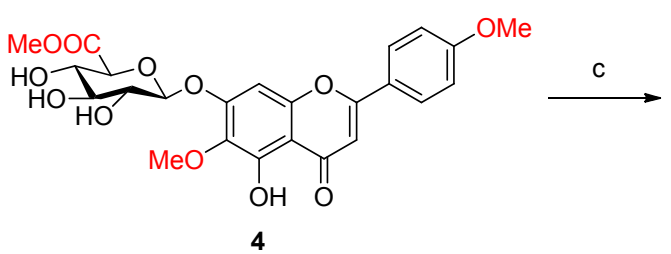<smiles>COc1ccc(-c2cc(=O)c3c(O)c(OC)c(O)cc3o2)cc1</smiles>

Reagents and conditions: (a) $\mathrm{SOCl}_{2}$ (10.0 equiv.), $\mathrm{MeOH}, 93 \%$; (b) Mel (2.4 equiv.), $\mathrm{K}_{2} \mathrm{CO}_{3}$ (3.0 equiv.), DMF, $84 \%$; (c) $\mathrm{H}_{2} \mathrm{SO}_{4}$, EtOH, reflux, 89\%

图式 1 柳穿鱼黄素的合成路线

Scheme 1 Synthesis of pectolinarigenin

得到糖基化受体柳穿鱼黄素 $\mathbf{5}^{[23]}$. 经过比对, 采用此法 合成的柳穿鱼黄素核磁共振图谱与天然产物数据一 致 ${ }^{[14 b]}$.

在成功制备得到糖基化受体柳穿鱼黄素 $\mathbf{5}$ 后, 我们 将合成的重点转移到芸香糖糖基供体 10 的制备上. 其 合成路线如 Scheme 2 所示. 我们以价廉易得的 $D$-葡萄 糖为原料, 将 6-位伯羟基经叔丁基二苯基硅基选择性保 护后, 剩余羟基全苯甲酰化保护, 两步一锅法以 $83 \%$ 的 收率制备得到化合物 7. 化合物 7 有 $\alpha 、 \beta$ 两种构型, 其 中 $\alpha$ 构型占比较小, $\beta$ 构型为主要产物, 两者可通过柱层 析实现完全分离. 以 $\beta$ 构型的化合物 7 为原料, 使用我 们课题组发展的以固体负载酸 $\mathrm{TfOH}-\mathrm{SiO}_{2}$ 催化选择性脱 除 6-位叔丁基二苯基硅基的新方法 ${ }^{[24]}$, 以 $89 \%$ 的收率得 到糖基受体 8 . 糖基受体 8 与 $L$-鼠李糖糖基供体 $\mathbf{1 2}^{[25]}$ 在促进剂三甲基硅基三氟甲烷磺酸酯的催化下发生糖 苷化反应，以 $67 \%$ 的收率制备得到双糖化合物 9. 化合 物 9 在乙酰溴一甲醇体系下可实现端基溴代, 以 $88 \%$ 的 收率得到全苯甲酰化的溴代芸香糖糖基供体 $\mathbf{1 0}$.

在成功制备得到糖基受体柳穿鱼黄素 $\mathbf{5}$ 以及芸香糖
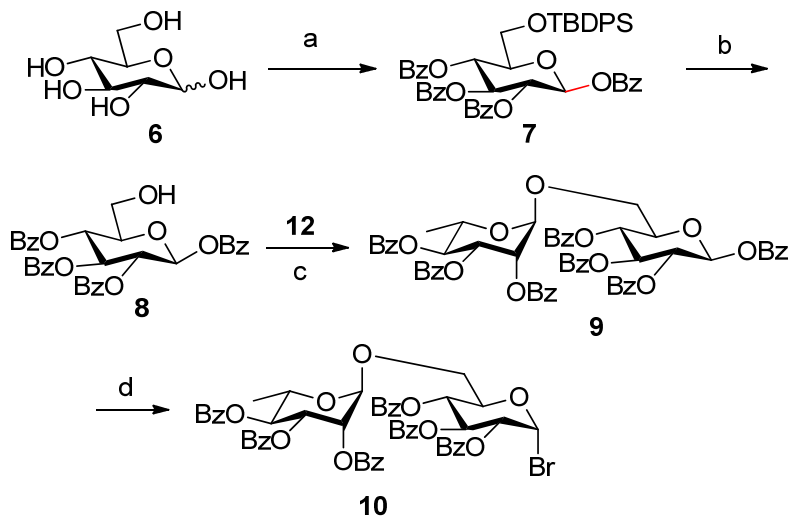

Reagents and conditions: (a) TBDPSCI (1.5 equiv.), Im (3.0 equiv.), $\mathrm{BzCl}$ (6.0 equiv.), $\mathrm{Py}, 73 \%$; (b) $\mathrm{TfOH}-\mathrm{SiO}_{2}$ (0.2 equiv.), $\mathrm{MeCN}, 89 \%$; (c) 12 (1.2 equiv.), TMSOTf (0.15 equiv.), 67\%; (d) $\mathrm{AcBr}(5.0$ equiv.), $\mathrm{MeOH}$ (3.0 equiv.), $\mathrm{AcOH}, 8 \mathrm{~h}, 88 \%$

图式 2 芸香糖糖基供体的合成路线

Scheme 2 Synthesis of benzobromorutinose donor

糖基供体 10 后, 我们首先试验了相转移催化下的糖苷 化反应. 在相转移催化剂四丁基溴化铵存在的条件下, 将柳穿鱼黄素 $\mathbf{5}$ 与糖基供体 $\mathbf{1 0}$ 首先室温摚拌下溶于氯 仿, 然后向反应体系中加入等体积的 $0.5 \mathrm{~mol} / \mathrm{L}$ 的碳酸 钾溶液 ${ }^{[26]}$. 令我们高兴的是 $45{ }^{\circ} \mathrm{C}$ 下加热反应 $12 \mathrm{~h}$ 后以 48\%的收率得到目标化合物前体 11 , 且反应中未检测到 有 5 -位糖苷化的产物生成. 当采用饱和碳酸钾溶液时, $45{ }^{\circ} \mathrm{C}$ 下加热反应 $18 \mathrm{~h}$, 能以 $62 \%$ 的收率得到化合物 11. 化合物 11 端基质子的化学位移为 $\delta=5.70$, 其偶合常数 $J=9.7 \mathrm{~Hz}$, 可以判断其为 $\beta$ 构型糖苷键. 核磁共振谱图 中未发现 $\alpha$ 构型糖苷键的存在. 黄酮糖苷化合物 11 在四 氢呋喃一甲醇-水混合溶剂体系中 ${ }^{[27]}$, 采用 2 equiv.的无 水碳酸钾可以顺利地脱除糖环上的保护基，以 $89 \%$ 的收 率得到需要的最终产物. 经过比对, 采用此法合成的柳 穿鱼叶苷核磁共振图谱与天然产物数据一致 ${ }^{[14 \mathrm{~b}]}$.

\section{2 结论}

柳穿鱼黄素及柳穿鱼叶苷作为中药大䔡中黄酮及 黄酮苷类化合物的代表，具有十分重要的生物活性. 本 文以商业易得的灯或花乙素为原料，经羧基酯化、酚羟 基选择性甲基化及糖苷键水解 3 步反应，以 $69.5 \%$ 的总 收率得到柳穿鱼黄素. 随后, 得到的柳穿鱼黄素为糖基 受体, 全苯甲酰基保护的溴代芸香糖为糖基供体完成了 柳穿鱼叶苷的化学合成. 终产物及中间体经 NMR 和 HRMS 结构确证. 该合成方法较之从植物中提取柳穿鱼 黄素及柳穿鱼叶苷具有原料易得、操作简单、可大量制 备等优点, 为后期深入研究它们的药理活性及结构改造 奠定了很好的物质基础. 

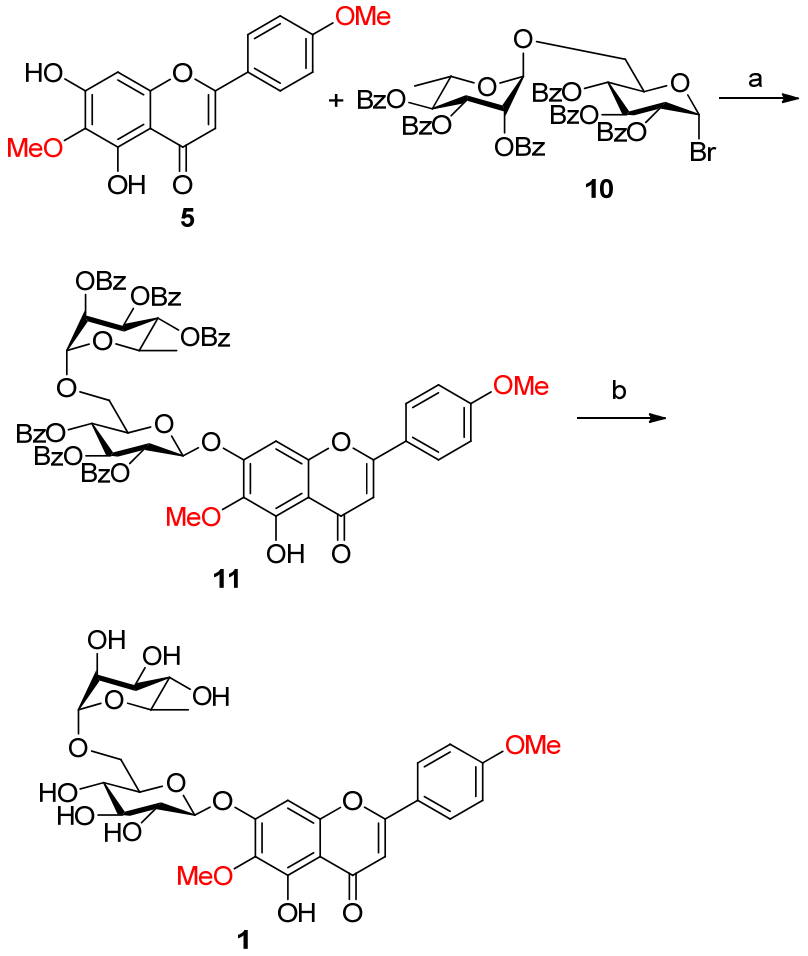

Reagents and conditions: (a) TBAB ( 0.5 equiv.), aq. $\mathrm{K}_{2} \mathrm{CO}_{3}, 45{ }^{\circ} \mathrm{C}$, $18 \mathrm{~h}, 62 \%$; (b) $\mathrm{K}_{2} \mathrm{CO}_{3}$ (2.0 equiv.), THF-MeOH- $\mathrm{H}_{2} \mathrm{O}, 2 \mathrm{~h}, 89 \%$

图 5 柳穿鱼叶苷的合成路线

Figure 5 Synthesis of pectolinarin

\section{3 实验部分}

\section{1 仪器与试剂}

熔点采用上海精密科学仪器有限公司制造 WRR 型 熔点仪测定; 核磁共振波谱采用 Bruker VANCE-400 或 600 型核磁共振仪测定; 质谱采用 Agilent 1946BESIMS 型质谱仪测定; 旋光采用 SGW-3 自动旋光仪测定. 薄层色谱(TLC)采用 $\mathrm{HSGF}_{254}$ (烟台化学工业研究所), 柱 层析使用硅胶为 $200 \sim 300$ 目 (烟台化学工业研究所). 所 用试剂和溶剂为市售化学纯或分析纯.

\section{2 实验方法}

\section{2 .1 灯盛花乙素甲酯(3)的合成}

冰浴下向 $200 \mathrm{~mL}$ 甲醇中缓慢滴加氯化亚砜(7.26 $\mathrm{mL}, 100.00 \mathrm{mmol})$, 滴加完毕撤去冰浴. 室温搅拌 $1 \mathrm{~h}$ 后 向反应体系中加入灯戞花乙素(2) (4.62 g, $10.00 \mathrm{mmol})$, 室温继续搅拌反应 $6 \mathrm{~h}$, 经薄层色谱 (TLC) [ $V($ 乙酸乙 酯)：V(异丙醇) $: V($ 水 $)=4: 2: 1$ ]检测显示反应完全. 减压浓缩得粗品, 将粗品悬浮于 $100 \mathrm{~mL}$ 乙酸乙酯, 加 热回流 $30 \mathrm{~min}$ 后, 自然冷却. 过滤, 滤饼用乙酸乙酯洗 涤, 真空干燥得黄色固体 $34.42 \mathrm{~g}$, 收率 93\%. m.p. $277 \sim 279{ }^{\circ} \mathrm{C} ;[\alpha]_{\mathrm{D}}^{25}-3.0$ (c 0.10, DMSO); ${ }^{1} \mathrm{H}$ NMR (400 MHz, DMSO- $\left.d_{6}\right) \delta: 12.75(\mathrm{~s}, 1 \mathrm{H}, 5-\mathrm{OH}), 10.39(\mathrm{~s}, 1 \mathrm{H}$,
4'-OH), 8.63 (s, 1H, 6-OH), 7.93 (d, $J=8.3 \mathrm{~Hz}, 2 \mathrm{H}, \mathrm{H}-2$ ', H-6'), 7.00 (s, 1H, H-8), 6.94 (d, $J=8.3 \mathrm{~Hz}, 2 \mathrm{H}, \mathrm{H}-3$ ', H-5'), 6.82 (s, 1H, H-3), 5.27 (d, $J=7.0 \mathrm{~Hz}, 1 \mathrm{H}, \mathrm{H}-1 ")$, 4.20 (d, $J=9.4 \mathrm{~Hz}, 1 \mathrm{H}, \mathrm{H}-2 "), 3.67$ (s, $\left.3 \mathrm{H}, \mathrm{OCH}_{3}\right) ;{ }^{13} \mathrm{C}$ NMR $\left(150 \mathrm{MHz}\right.$, DMSO- $d_{6}$ ) $\delta: 182.36,169.22,164.13$, $161.21,150.96,149.01,146.92,130.46,128.47,121.31$, $116.00,105.89,102.56,99.84,93.52,75.28,75.04,72.76$, 71.41, $52.02\left(\mathrm{OCH}_{3}\right)$; IR (KBr) v: 3581, 3428, 2027, 1740, 1660, 1577, 1503, 1453, 1353, 1240, 1191, 1084, 830, 744, $586 \mathrm{~cm}^{-1}$; HRMS calcd for $\mathrm{C}_{22} \mathrm{H}_{21} \mathrm{O}_{12} 477.1033$, found 477.1026 .

\section{$3.2 .26,4^{\prime}$-二甲氧基灯盛花乙素甲酯(4)的合成}

将灯盏花乙素甲酯(3) (1.61 g, $3.38 \mathrm{mmol})$ 悬浮于无 水 DMF $25 \mathrm{~mL}$, 氮气保护下加入无水碳酸钾 $(1.40 \mathrm{~g}$, $10.14 \mathrm{mmol})$, 室温搅拌 $10 \mathrm{~min}$ 后注入碘甲烷 $(0.50 \mathrm{~mL}$, $8.11 \mathrm{mmol})$. 室温搅拌过夜, TLC [ $V$ (二氯甲烷) $: V$ (甲 醇 $)=10 ：$ ] 检测显示反应完全. 将反应液倾入 $1 \mathrm{~mol} / \mathrm{L}$ 盐酸 $50 \mathrm{~mL}$ 中, 乙酸乙酯萃取 $(50 \mathrm{~mL} \times 2)$, 合并有机相, 饱和食盐水 $100 \mathrm{~mL}$ 洗涤, 无水硫酸钠干燥. 减压浓缩 柱层析得黄色固体 $41.43 \mathrm{~g}$, 收率 84\%. m.p. 256 $259{ }^{\circ} \mathrm{C} ;[\alpha]_{\mathrm{D}}^{25}-24.6$ (c 0.12, DMSO); ${ }^{1} \mathrm{H}$ NMR (400 MHz, DMSO- $\left.d_{6}\right) \delta: 12.95$ (s, $\left.1 \mathrm{H}, 5-\mathrm{OH}\right), 8.06$ (d, $J=8.8$ Hz, 2H, H-2', H-6'), 7.15 (d, $J=8.8$ Hz, 2H, H-3', H-5'), 7.11 (s, 1H, H-3), 6.97 (s, 1H, H-8), 5.68 (d, $J=4.0 \mathrm{~Hz}$, $1 \mathrm{H}), 5.58(\mathrm{~d}, J=5.1 \mathrm{~Hz}, 1 \mathrm{H}), 5.45(\mathrm{~d}, J=3.5 \mathrm{~Hz}, 1 \mathrm{H}), 5.39$ (d, $J=6.7 \mathrm{~Hz}, 1 \mathrm{H}, \mathrm{H}-1 "), 4.23$ (d, $J=9.2 \mathrm{~Hz}, 1 \mathrm{H}, \mathrm{H}-2 ")$, $3.87\left(\mathrm{~s}, 3 \mathrm{H}, \mathrm{OCH}_{3}\right), 3.77\left(\mathrm{~s}, 3 \mathrm{H}, \mathrm{OCH}_{3}\right), 3.67(\mathrm{~s}, 3 \mathrm{H}$, $\mathrm{OCH}_{3}$ ), $3.48 \sim 3.40$ (m, 3H, H-3", H-4", H-5"); ${ }^{13} \mathrm{C}$ NMR $\left(150 \mathrm{MHz}, \mathrm{DMSO}-d_{6}\right) \delta: 182.38,169.21,163.92,162.49$, $156.01,152.64,152.17,132.55,128.40,122.74,114.65$, 105.97, 103.43, 99.38, 93.97, 75.70, 75.26, 72.82, 71.34, 60.32, 55.63, 52.00; IR (KBr) v: 3569, 3517, 3362, 2912, 1741, 1664, 1466, 1365, 1304, 833, $582 \mathrm{~cm}^{-1}$; HRMS calcd for $\mathrm{C}_{24} \mathrm{H}_{25} \mathrm{O}_{12} 505.1346$, found 505.1337.

\subsection{3 柳穿鱼黄素(5)的合成}

将化合物 $4(1.51 \mathrm{~g}, 3.00 \mathrm{mmol})$ 悬浮于 $60 \mathrm{~mL}$ 乙醇及 $10 \mathrm{~mL}$ 水的混合溶剂中, 室温剧烈搅拌下缓慢滴加 10 $\mathrm{mL}$ 浓硫酸. 滴加完毕 $110{ }^{\circ} \mathrm{C}$ 下加热反应 $10 \mathrm{~h}$, 经 TLC $[V($ 二氯甲烷 $): V($ 甲醇 $)=10 ： 1]$ 检测显示反应完全. 停 止加热, 自然冷却至室温析出固体, 过滤真空干燥得黄 色固体 $50.84 \mathrm{~g}$, 收率 89\%. m.p. 216 219 ${ }^{\circ} \mathrm{C} ;{ }^{1} \mathrm{H}$ NMR (400 MHz, DMSO- $\left.d_{6}\right) \delta: 13.04(\mathrm{~s}, 1 \mathrm{H}, 5-\mathrm{OH}), 10.74(\mathrm{~s}$, $1 \mathrm{H}, 7-\mathrm{OH}), 8.03$ (d, $\left.J=8.6 \mathrm{~Hz}, 2 \mathrm{H}, \mathrm{H}-2^{\prime}, \mathrm{H}-6^{\prime}\right), 7.10$ (d, $\left.J=8.6 \mathrm{~Hz}, 2 \mathrm{H}, \mathrm{H}-3^{\prime}, \mathrm{H}-5^{\prime}\right), 6.87$ (s, 1H, H-3), 6.62 (s, 1H, $\mathrm{H}-8), 3.86\left(\mathrm{~s}, 3 \mathrm{H}, \mathrm{OCH}_{3}\right), 3.76\left(\mathrm{~s}, 3 \mathrm{H}, \mathrm{OCH}_{3}\right) ;{ }^{13} \mathrm{C} \mathrm{NMR}$ 
(150 MHz, DMSO- $\left.d_{6}\right) \delta: 182.17$ (C-4), 163.36 (C-2), 162.30 (C-4'), 157.34 (C-7), 152.78 (C-9), 152.43 (C-5), 131.38 (C-6), 128.29 (C-2', C-6'), 122.86 (C-1'), 114.56 (C-3', C-5'), 104.16 (C-10), 103.06 (C-3), 94.31 (C-8), 59.97 (OMe), 55.55 (OMe); IR (KBr) v: 3924, 3811, 3600, 3457, 3369, 1660, 1620, 1470, 1368, 1274, 1176, 1101 $\mathrm{cm}^{-1}$; HRMS calcd for $\mathrm{C}_{17} \mathrm{H}_{14} \mathrm{O}_{6} 314.0790$, found 314.0782 .

3.2.4 2,3,4-三-( $O$ - 苯甲酰基)-6- $O$-叔丁基二苯基硅 基- $D$-吡喃葡萄糖苯甲酰基苷(7)的合成

将 $D$-葡萄糖 $6(3.60 \mathrm{~g}, 20.00 \mathrm{mmol})$ 溶于无水吡定 $100 \mathrm{~mL}$, 氮气保护下依次加入咪坐 $(4.08 \mathrm{~g}, 60.00 \mathrm{mmol})$ 及叔丁基二苯基氯硅烷(TBDPSCl, $7.80 \mathrm{~mL}, 30.00$ $\mathrm{mmol})$. 室温摚拌 $2 \mathrm{~h}$ 后, 冰浴下缓慢滴加苯甲酰氯 (14.03 mL, $120.00 \mathrm{mmol})$, 滴加完毕后撤去冰浴, 向反 应体系中加入 4-二甲氨基吡啶(DMAP, $0.24 \mathrm{~g}, 2.00$ $\mathrm{mmol}$ ), $40{ }^{\circ} \mathrm{C}$ 下加热反应 $8 \mathrm{~h}$. 经 TLC [ $V$ (石油醚) $: V($ 乙 酸乙酯) $=2: 1$ ] 检测显示反应完全. 加入甲醇 $10 \mathrm{~mL}$ 终 止反应, 减压蒸除大部分溶剂后将剩余液倾入 $1 \mathrm{~mol} / \mathrm{L}$ 盐酸 $300 \mathrm{~mL}$ 中, 二氯甲烷萃取 $(100 \mathrm{~mL} \times 2)$, 合并有机 相, 饱和食盐水 $100 \mathrm{~mL}$ 洗涤, 无水硫酸钠干燥. 减压浓 缩柱层析得白色泡沫状固体 $712.19 \mathrm{~g}$, 收率 $73 \%$. For $\beta$ isomer m.p. $74 \sim 76{ }^{\circ} \mathrm{C}$; $[\alpha]_{\mathrm{D}}^{25}+19.0\left(c 0.10, \mathrm{CHCl}_{3}\right)$; ${ }^{1} \mathrm{H}$ NMR $\left(400 \mathrm{MHz}, \mathrm{CDCl}_{3}\right) \delta: 8.09(\mathrm{~d}, J=7.5 \mathrm{~Hz}, 2 \mathrm{H}$, Ph-H), 7.90 (dd, $J=17.2,8.2 \mathrm{~Hz}, 6 \mathrm{H}, \mathrm{Ph}-\mathrm{H}$ ), 7.66 (d, $J=$ $6.9 \mathrm{~Hz}, 2 \mathrm{H}, \mathrm{Ph}-\mathrm{H}), 7.56$ (dd, $J=18.3,7.5 \mathrm{~Hz}, 4 \mathrm{H}, \mathrm{Ph}-\mathrm{H}$ ), $7.49 \sim 7.28(\mathrm{~m}, 12 \mathrm{H}, \mathrm{Ph}-\mathrm{H}), 7.23(\mathrm{~d}, J=7.0 \mathrm{~Hz}, 2 \mathrm{H}$, Ph-H), 7.12 (t, $J=7.5 \mathrm{~Hz}, 2 \mathrm{H}, \mathrm{Ph}-\mathrm{H}), 6.25$ (d, $J=7.9 \mathrm{~Hz}$, $1 \mathrm{H}, \mathrm{H}-1), 5.95$ (t, $J=9.4 \mathrm{~Hz}, 1 \mathrm{H}, \mathrm{H}-2), 5.83$ (dt, $J=12.4$, $9.3 \mathrm{~Hz}, 2 \mathrm{H}), 4.08(\mathrm{~d}, J=9.7 \mathrm{~Hz}, 1 \mathrm{H}), 3.88(\mathrm{dt}, J=12.0,7.1$ $\mathrm{Hz}, 2 \mathrm{H}), 1.01$ (s, 9H, H-t-Bu); ${ }^{13} \mathrm{C}$ NMR (150 MHz, $\left.\mathrm{CDCl}_{3}\right) \delta: 165.95,165.27,165.08,164.80,135.84,135.71$, $133.89,133.49,133.44,133.36,133.14,133.08,130.32$, $129.97,129.73,129.69,129.28,129.05,128.92,128.70$, $128.53,128.46,127.71,127.66,92.84,75.87,73.42$, $71.25,68.79,62.52,26.78,19.32$; IR (KBr) $v: 3963,3740$, 3441, 3062, 2938, 1766, 1736, 1598, 1456, 1268, 1081, 1026, $704 \mathrm{~cm}^{-1}$; HRMS calcd for $\mathrm{C}_{50} \mathrm{H}_{47} \mathrm{O}_{10} \mathrm{Si} 835.2938$, found 835.2927.

3.2.5 $2,3,4$-三-( $O$ - 苯甲酰基 $)-\beta$ - $D$ - 吡喃葡萄糖苯甲酰 基苷 $(8)$ 的合成

将化合物 7 (4.18 g, $5.00 \mathrm{mmol})$ 溶于乙腈 $50 \mathrm{~mL}$, 室 温搅拌下加入 $2.0 \mathrm{mmol} / \mathrm{g}$ TfOH-SiO $2(0.50 \mathrm{~g}, 1.00$ mmol). $60{ }^{\circ} \mathrm{C}$ 下加热反应 $1 \mathrm{~h}$ 后 TLC [ $V$ (石油梄) $: V($ 乙
酸乙酯) $=3: 1]$ 检测显示反应完全. 过滤, 乙腈洗涤, 减压浓缩柱层析得白色泡沫状固体 $82.65 \mathrm{~g}$, 收率 89\%. m.p. $159 \sim 161{ }^{\circ} \mathrm{C} ;[\alpha]_{\mathrm{D}}^{25}+0.2\left(c 0.10, \mathrm{CHCl}_{3}\right) ;{ }^{1} \mathrm{H}$ NMR $\left(400 \mathrm{MHz}, \mathrm{CDCl}_{3}\right) \delta: 8.04$ (d, J=7.6 Hz, 2H, Ph-H), 7.97 (d, $J=7.5 \mathrm{~Hz}, 2 \mathrm{H}, \mathrm{Ph}-\mathrm{H}), 7.89$ (dd, $J=15.1,7.7 \mathrm{~Hz}, 4 \mathrm{H}$, Ph-H), 7.56 (s, 2H, Ph-H), 7.44 (dd, $J=16.0,7.9$ Hz, 6H, Ph-H), $7.37 \sim 7.28$ (m, 4H, Ph-H), 6.25 (d, $J=7.8 \mathrm{~Hz}, 1 \mathrm{H}$, H-1), 6.09 (t, $J=9.8 \mathrm{~Hz}, 1 \mathrm{H}, \mathrm{H}-3), 5.85(\mathrm{t}, J=8.8 \mathrm{~Hz}, 1 \mathrm{H}$, $\mathrm{H}-2), 5.62$ (t, $J=9.6 \mathrm{~Hz}, 1 \mathrm{H}, \mathrm{H}-4), 4.02(\mathrm{~d}, J=9.6 \mathrm{~Hz}$, 1H), $3.92(\mathrm{~s}, 1 \mathrm{H}), 3.75(\mathrm{~d}, J=12.4 \mathrm{~Hz}, 1 \mathrm{H}), 2.68(\mathrm{~s}, 1 \mathrm{H}$, 6-OH).; ${ }^{13} \mathrm{C}$ NMR $\left(150 \mathrm{MHz}, \mathrm{CDCl}_{3}\right) \delta: 166.24,165.83$, $165.21,164.92,133.99,133.93,133.54,133.48,130.31$, $130.10,129.89,129.87,128.89,128.84,128.83,128.66$, $128.52,128.48,92.88,75.71,72.76,70.95,69.20,61.06$; IR (KBr) v: 3859, 3742, 3540, 3065, 1732, 1598, 1262, 1087, $707 \mathrm{~cm}^{-1}$; HRMS calcd for $\mathrm{C}_{34} \mathrm{H}_{29} \mathrm{O}_{10} 597.1761$, found 597.1767.

3.2.6 2,3,4-三- $(O$ - 苯甲酰基 $)-\alpha-L$ - 鼠李糖甘-( $1 \rightarrow 6)$ $2,3,4$-三- $O$ - 苯甲酰基- $\beta$ - $D$ - 吡喃葡萄糖苯甲酰基苷 $(9)$ 的合成

将糖基受体 8 (2.31 g, $3.86 \mathrm{mmol})$ 及全苯甲酰基保 护的 $L$-鼠李糖三氯乙酰亚胺酯糖基供体 $\mathbf{1 2}(2.87 \mathrm{~g}, 4.63$ $\mathrm{mmol}$ )溶于无水二氯甲烷 $60 \mathrm{~mL}$, 氮气保护下下加入 $4 \AA$ 分子篮 $2.0 \mathrm{~g}$. 室温搅拌 $30 \mathrm{~min}$ 后, 冰浴下注入三氟甲磺 酸三甲基硅酯(TMSOTf, $0.11 \mathrm{~mL}, 0.38 \mathrm{mmol}), 2 \mathrm{~h}$ 后 TLC $[V$ (石油醚) $: V($ 乙酸乙酯 $)=3: 1]$ 检测显示反应完 全. 向反应体系中加入 $0.5 \mathrm{~mL}$ 三乙胺终止反应, 减压浓 缩柱层析得白色泡沫状固体 $92.72 \mathrm{~g}$, 收率 67\%. m.p. $115 \sim 118{ }^{\circ} \mathrm{C} ;[\alpha]_{\mathrm{D}}^{25}+69.5\left(c \quad 0.20, \mathrm{CHCl}_{3}\right) ;{ }^{1} \mathrm{H}$ NMR $\left(400 \mathrm{MHz}, \mathrm{CDCl}_{3}\right) \delta: 8.04 \sim 7.77(\mathrm{~m}, 15 \mathrm{H}, \mathrm{Ph}-\mathrm{H}), 7.60 \sim$ 7.28 (m, 20H, Ph-H), 6.26 (d, J=8.0 Hz, 1H, H-1), 6.05 (t, $J=9.6 \mathrm{~Hz}, 1 \mathrm{H}), 5.92 \sim 5.78(\mathrm{~m}, 2 \mathrm{H}), 5.74\left(\mathrm{~s}, 1 \mathrm{H}, \mathrm{H}-1^{\prime}\right)$, $5.63(\mathrm{dt}, J=19.9,9.8 \mathrm{~Hz}, 2 \mathrm{H}), 5.10(\mathrm{~s}, 1 \mathrm{H}), 4.37(\mathrm{~s}, 1 \mathrm{H})$, $4.31 \sim 4.22(\mathrm{~m}, 1 \mathrm{H}), 4.01 \sim 3.97(\mathrm{~m}, 2 \mathrm{H}), 1.30(\mathrm{~d}, J=6.1$ $\left.\mathrm{Hz}, 3 \mathrm{H}, \mathrm{H}-6{ }^{\prime}\right) ;{ }^{13} \mathrm{C}$ NMR $\left(150 \mathrm{MHz}, \mathrm{CDCl}_{3}\right) \delta: 165.95$, $165.84,165.48,165.39,165.34,165.20,164.72,133.75$, $133.52,133.46,133.39,133.36,133.09,130.33,130.24$, $130.08,130.04,129.67,129.53,129.50,128.96,128.87$, 128.76, 128.71, 128.66, 128.60, 128.56, 128.53, 128.49, $128.34,98.85,92.90,75.25,72.98,71.99,70.68,69.98$, 69.50, 67.35, 67.08, 17.80; IR (KBr) v: 3750, 3657, 3530, 3435, 3368, 3151, 3067, 2975, 1732, 1601, 1450, 1263, 1099, 711, $586 \mathrm{~cm}^{-1}$; HRMS calcd for $\mathrm{C}_{61} \mathrm{H}_{51} \mathrm{O}_{17}$ 1055.3126, found 1055.3118 . 
3.2.7 2,3,4-三-( $O$ - 苯甲酰基) - $\alpha$ - $L$ - 鼠李糖苷-( $1 \rightarrow 6)$ $2,3,4$-三-( $O$ - 苯甲酰基) $-\alpha-D$-吡喃葡萄糖溴苷 $(\mathbf{1 0})$ 的合 成

将化合物 9 (1.06 g, $1.00 \mathrm{mmol})$ 溶于冰醋酸 $10 \mathrm{~mL}$, 室温氮气保护下依次加入乙酰溴 $(0.37 \mathrm{~mL}, 5.00 \mathrm{mmol})$ 及甲醇 $(0.12 \mathrm{~mL}, 3.00 \mathrm{mmol})$, 室温搅拌 $8 \mathrm{~h}$ 后 TLC [ $V$ (石 油醚) $: V($ 乙酸乙酯 $)=4: 1]$ 检测显示反应完全. 向反应 体系中加入二氯甲烷 $30 \mathrm{~mL}$ 稀释, 分别用冷水、饱和碳 酸氢钠水溶液、水各 $20 \mathrm{~mL}$ 洗涤, 无水硫酸钠干燥, 减 压浓缩柱层析得白色固体 $100.88 \mathrm{~g}$, 收率 88\%. m.p. $169 \sim 172{ }^{\circ} \mathrm{C} ;[\alpha]_{\mathrm{D}}^{25}+115.0\left(c \quad 0.10, \mathrm{CHCl}_{3}\right) ;{ }^{1} \mathrm{H} \mathrm{NMR}$ $\left(400 \mathrm{MHz}, \mathrm{CDCl}_{3}\right) \delta: 8.09$ (d, J=7.6 Hz, 2H, Ph-H), 8.00 (dt, $J=16.0,8.1 \mathrm{~Hz}, 6 \mathrm{H}, \mathrm{Ph}-\mathrm{H}), 7.92$ (d, $J=7.6 \mathrm{~Hz}, 2 \mathrm{H}$, Ph-H), 7.82 (d, J=7.6 Hz, 2H, Ph-H), 7.64 7.27 (m, $18 \mathrm{H}, \mathrm{Ph}-\mathrm{H}), 6.96$ (d, $J=3.8 \mathrm{~Hz}, 1 \mathrm{H}, \mathrm{H}-1), 6.29$ (t, $J=9.8$ $\mathrm{Hz}, 1 \mathrm{H}, \mathrm{H}-3), 5.90 \sim 5.73(\mathrm{~m}, 3 \mathrm{H}), 5.65$ (t, $J=9.9 \mathrm{~Hz}, 1 \mathrm{H})$, $5.36(\mathrm{dd}, J=9.9,3.9 \mathrm{~Hz}, 1 \mathrm{H}), 5.00$ (s, 1H, H-1'), $4.69 \sim$ $4.56(\mathrm{~m}, 1 \mathrm{H}), 4.21(\mathrm{dd}, J=9.5,6.3 \mathrm{~Hz}, 1 \mathrm{H}), 4.03$ (d, $J=$ $11.9 \mathrm{~Hz}, 1 \mathrm{H}), 3.84$ (dd, $J=12.1,5.0 \mathrm{~Hz}, 1 \mathrm{H}), 1.33$ (d, $J=$ $6.2 \mathrm{~Hz}, 3 \mathrm{H}, \mathrm{H}-6 ') ;{ }^{13} \mathrm{C} \mathrm{NMR}\left(150 \mathrm{MHz}, \mathrm{CDCl}_{3}\right) \delta: 165.96$, $165.76,165.50,165.45,165.39,165.21,133.87,133.76$, $133.53,133.48,133.16,130.24,130.14,130.07,129.95$, $129.85,129.61,129.42,128.99,128.68,128.57,128.50$, $128.37,98.81,87.20,74.12,71.92,71.71,69.96,68.19$, 67.20, 66.59, 17.79; IR (KBr) v: 3920, 3747, 3531, 3091, 2978, 1730, 1602, 1452, 1268, 1105, $711 \mathrm{~cm}^{-1}$; HRMS calcd for $\mathrm{C}_{54} \mathrm{H}_{46} \mathrm{BrO}_{15}$ 1013.2020, found 1013.2013.

$3.2 .82,3,4$-三- $(O$ - 苯甲酰基 $)-\alpha-L$ - 鼠李糖苷- $(1 \rightarrow 6)-$ $2,3,4$-三-( $O$-苯甲酰基) $-\beta$ - $D$-吡喃葡萄糖柳穿鱼黄素基 苷(11)的合成

将化合物 5 (0.49 g, $1.56 \mathrm{mmol})$, 化合物 10 (2.37 g, $2.34 \mathrm{mmol}$ ) 溶于氯仿 $30 \mathrm{~mL}$, 室温搅拌下依次加入四丁 基溴化铵 $(0.25 \mathrm{~g}, 0.78 \mathrm{mmol})$ 及饱和碳酸钾溶液 $30 \mathrm{~mL}$. 室温搅拌 $10 \mathrm{~min}, 45^{\circ} \mathrm{C}$ 下加热反应 $18 \mathrm{~h}$ 后 TLC [ $V($ 石油 醚) $: V($ 乙酸乙酯 $)=4: 1]$ 检测显示反应完全. 分液, 水 相用二氯甲烷萃取 $(20 \mathrm{~mL} \times 2)$, 合并有机相, 饱和食盐 水 $50 \mathrm{~mL}$ 洗涤, 无水硫酸钠干燥. 减压浓缩柱层析得白 色固体 $111.20 \mathrm{~g}$, 收率 62\%. m.p. 177 $179{ }^{\circ} \mathrm{C}$; $[\alpha]_{\mathrm{D}}^{25}$ -29.0 (c 0.10, $\mathrm{CHCl}_{3}$ ); ${ }^{1} \mathrm{H}$ NMR (400 MHz, $\left.\mathrm{CDCl}_{3}\right) \delta$ : $12.88(\mathrm{~s}, 1 \mathrm{H}, 5-\mathrm{OH}), 8.02 \sim 7.98(\mathrm{~m}, 6 \mathrm{H}, \mathrm{Ph}-\mathrm{H}), 7.93$ (dd, $J=11.7,7.8 \mathrm{~Hz}, 4 \mathrm{H}, \mathrm{Ph}-\mathrm{H}), 7.71$ (d, $J=8.8 \mathrm{~Hz}, 2 \mathrm{H}$, $\mathrm{Ph}-\mathrm{H}), 7.65$ (d, $J=7.6 \mathrm{~Hz}, 2 \mathrm{H}, \mathrm{Ph}-\mathrm{H}), 7.58$ (t, $J=7.3 \mathrm{~Hz}$, $1 \mathrm{H}, \mathrm{Ph}-\mathrm{H}), 7.53 \sim 7.42(\mathrm{~m}, 6 \mathrm{H}, \mathrm{Ph}-\mathrm{H}), 7.40 \sim 7.31(\mathrm{~m}, 9 \mathrm{H}$, Ph-H), 7.19 (t, J=7.7 Hz, 2H, Ph-H), 6.92 (s, 1H, H-3), 6.79 (d, J=8.8 Hz, 2H, H-3', H-5'), 6.48 (s, 1H, H-8), 6.06 $(\mathrm{t}, J=9.3 \mathrm{~Hz}, 1 \mathrm{H}), 5.98 \sim 5.87(\mathrm{~m}, 1 \mathrm{H}), 5.77 \sim 5.68(\mathrm{~m}$, 2H), 5.66 (s, 1H, H-1"'), 5.61 (dd, $J=11.8,8.6 \mathrm{~Hz}, 2 \mathrm{H})$, $5.09(\mathrm{~s}, 1 \mathrm{H}), 4.39 \sim 4.30(\mathrm{~m}, 1 \mathrm{H}), 4.16 \sim 4.05(\mathrm{~m}, 2 \mathrm{H})$, $3.94(\mathrm{dd}, J=11.5,6.4 \mathrm{~Hz}, 1 \mathrm{H}), 3.66$ (s, 3H, OMe), 3.63 (s, $3 \mathrm{H}, \mathrm{OMe}), 1.20$ (d, $J=6.2 \mathrm{~Hz}, 3 \mathrm{H}, \mathrm{H}-6 " ') ;{ }^{13} \mathrm{C} \mathrm{NMR}(150$ $\left.\mathrm{MHz}, \mathrm{CDCl}_{3}\right) \delta: 183.02,165.85,165.38,165.28,165.18$, $164.77,162.58,155.26,154.10,152.44,134.66,133.75$, $133.55,133.51,133.46,133.04,130.04,129.97,129.94$, $129.83,129.69,129.34,129.32,129.18,128.82,128.80$, $128.67,128.54,128.51,128.21,128.15,123.34,114.46$, $108.28,103.99,100.06,98.02,97.37,74.55,72.54,71.76$, $71.70,70.65,69.91,69.61,67.09,66.89,61.05,55.33$, 17.59; IR (KBr) v: 3920, 3747, 3531, 3460, 3417, 3091, 2978, 1730, 1656, 1611, 1572, 1500, 1461, 1360, 1299, 1254, 1185, 1072, 833, 587, $711 \mathrm{~cm}^{-1}$; HRMS calcd for $\mathrm{C}_{71} \mathrm{H}_{59} \mathrm{O}_{21}$ 1247.3549, found 1247.3518.

\section{2 .9 柳穿鱼叶苷 $(\mathbf{1})$ 的合成}

将化合物 11 (280 mg, $0.22 \mathrm{mmol}$ )溶于甲醇 $5 \mathrm{~mL}$ 、 四氢呋喃 $5 \mathrm{~mL}$ 以及水 $1 \mathrm{~mL}$ 的混合溶剂中, 室温搅拌下 加入无水碳酸钾 $(62 \mathrm{mg}, 0.45 \mathrm{mmol})$. 室温搅拌反应 5 $\min$, 加热至 $45{ }^{\circ} \mathrm{C}$ 继续反应 $2 \mathrm{~h}$ 后 TLC [ $V$ (二氯甲烷)： $V($ 甲醇 $)=6: 1]$ 检测显示反应完全. 加入阳离子树脂中 和至中性, 过滤, 甲醇洗涤, 有机相减压浓缩得粗品, 将所得粗品悬浮于 $10 \mathrm{~mL}$ 乙酸乙酯, 加热回流 $30 \mathrm{~min}$ 后, 自然冷却, 过滤, 滤饼用乙酸乙酯洗涤, 真空干燥 得淡黄色固体 $1124 \mathrm{mg}$, 收率 89\%. m.p. 275 277 ${ }^{\circ} \mathrm{C}$; $[\alpha]_{\mathrm{D}}^{25}-45.0$ (c 0.28 , DMSO); ${ }^{1} \mathrm{H}$ NMR $(400 \mathrm{MHz}$, DMSO- $\left.d_{6}\right) \delta: 8.04$ (d, $J=8.5$ Hz, 2H, H-2', H-6'), 7.17 (d, $J=8.6 \mathrm{~Hz}, 2 \mathrm{H}, \mathrm{H}-3^{\prime}, \mathrm{H}-5$ '), 6.95 (s, 2H, H-3, H-8), 5.13 (d, $J=6.3 \mathrm{~Hz}, 1 \mathrm{H}, \mathrm{H}-1 "), 4.56$ (s, 1H, H-1"'), $3.89 \sim 3.86$ (m, $1 \mathrm{H}), 3.85$ (s, 3H, OMe), $3.76(\mathrm{~s}, 3 \mathrm{H}, \mathrm{OMe}), 3.69 \sim 3.60$ $(\mathrm{m}, 2 \mathrm{H}), 3.47(\mathrm{dd}, J=10.2,6.9 \mathrm{~Hz}, 2 \mathrm{H}), 3.43 \sim 3.37(\mathrm{~m}$, $1 \mathrm{H}), 3.35 \sim 3.27(\mathrm{~m}, 2 \mathrm{H}), 3.16(\mathrm{dd}, J=19.9,10.3 \mathrm{~Hz}, 2 \mathrm{H})$, $1.05\left(\mathrm{~d}, J=6.0 \mathrm{~Hz}, 3 \mathrm{H}, \mathrm{H}-6{ }^{\prime \prime}\right) ;{ }^{13} \mathrm{C} \mathrm{NMR}(150 \mathrm{MHz}$, DMSO- $\left.d_{6}\right) \delta: 182.22$ (C-4), 163.94 (C-2), 162.25 (C-4'), 159.39 (C-7), 152.39 (C-9), 152.05 (C-5), 132.53 (C-6), 128.29 (C-2', C-6'), 122.57 (C-1'), 114.62 (C-3', C-5'), 105.75 (C-10), 103.23 (C-3), 100.24 (C-1"'), 100.20 (C-1"), 94.19 (C-8), 76.30 (C-3"), 75.60 (C-5"), 73.00 (C-2"), 71.85 (C-4"'), 70.61 (C-3"'), 70.28 (C-2"'), 69.35 (C-4"), 68.17 (C-5"'), 65.80 (C-6"), 60.18 (6-OMe), 55.41 (4'-OMe), 17.61 (C-6"'); IR (KBr) v: 3460, 3417, 2932, 1656, 1611, 1572, 1500, 1461, 1360, 1299, 1254, 1185, $1072,833,587 \mathrm{~cm}^{-1}$; HRMS calcd for $\mathrm{C}_{29} \mathrm{H}_{35} \mathrm{O}_{15}$ 623.1976 , found 623.1987 . 
辅助材料(Supporting Information) 中间体及终产物 的 ${ }^{1} \mathrm{H} N \mathrm{NR}$ 及 ${ }^{13} \mathrm{C}$ NMR 谱图. 这些材料可以免费从本刊 网站(http://sioc-journal.cn/)上下载.

\section{References}

[1] Morimoto, M.; Tanimoto, K.; Nakano, S.; Ozaki, T.; Nakano, A.; Komai, K. J. Agric. Food Chem. 2003, 51, 389.

[2] Bandele, O. J.; Osheroff, N. Biochemistry 2007, 46, 6097.

[3] Daskiewicz, J. B.; Depeint, F.; Viornery, L.; Bayet, C.; Comte-Sarrazin, G.; Comte, G.; Gee, J. M.; Johnson, I. T.; Ndjoko, K. K.; Hostettmann, K.; Barron, D. J. Med. Chem. 2005, 48, 2790.

[4] Meragelman, T. L.; Tucker, K. D.; McClord, T. G.; Cardel-lina, J. H.; Shoemker, R. H. J. Nat. Prod. 2005, 68, 1790.

[5] Na, M.; Jang, J.; Njamen, D.; Mbafor, J. T.; Fomum, Z. T.; Kim, B. Y.; Oh, W. K.; Ahn, J. S. J. Nat. Prod. 2006, 69, 1572.

[6] Prestos, C.; Boziaris, I. S.; Nychas, J. E. Food Chem. 2005, 93, 1998.

[7] Yoon, H.; Kim, T. W.; Shin, S. Y.; Park, M. J.; Yong, Y.; Kim, D. W.; Islam, T.; Lee, Y. H.; Jung, K. Y.; Lim, Y. Biol. Med. Chem. Lett. 2013, 23, 232.

[8] Rahman, M. M.; Gray, A. I.; Khondkar, P.; Sarker, S. D. Pharm. Biol. 2008, 46, 356 .

[9] Hirpara, K. V.; Aggarwal, A. J.; Mukherjee, N.; Joshi; Burman, A. C. Curr. Med. Chem. 2009, 9, 138

[10] Nawghare, B.; Funde, S.; Raheem, A.; Lokhande, P. Chin. J. Chem. 2012, 30, 1695

[11] Walle, T.; Vincent, T. S.; Walle, U. K. Biochem. Pharmacol. 2003, 65,1603 .

[12] Tang, L.-J.; Zang, S.-F.; Yang, J.-Z.; Gao, W.-T. Chin. J. Org. Chem. 2004, 24, 882 (in Chinese).

(汤立军，张淑芬，杨锦宗，高文涛，有机化学, 2004, 24, 882.)
[13] Ishida, H.; Umino, T.; Tsuji, K.; Kosuge, T. Chem. Pharm. Bull. 1987, 35, 861 .

[14] (a) Park, J. C.; Lee, J. H.; Choi, J. S. Phytochemistry 1995, 39, 261 (b) Lim, H.; Son, K. H.; Chang, H. W.; Bae, K.; Kang, S. S.; Kim, H. P. Biol. Pharm. Bull. 2008, 31, 2063.

[15] Liu, S. J.; Luo, X.; Li, D. X.; Zhang, J.; Qiu, D. L.; Liu, W.; She, L.; Yang, Z. R. Int. Immunopharmacol. 2006, 6, 1387.

[16] Yoo, Y. M.; Nam, J. H.; Kim, M. Y.; Choi, J.; Park, H. J. Biol. Pharm. Bull. 2008, 31, 760.

[17] Lee, S.; Lee, D. H.; Kim, J. C.; Um, B. H.; Sung, S. H.; Jeong, L. S.; Kim, Y. K.; Kim, S. N. Biochem. Biophys. Res. Commun. 2017 493, 765 .

[18] Thamere, C.; Mourad, H.; Amel, B.; Nadir, B.; Ines, M.; Ramdane, S.; Souad, A.; Ahmed, M.; Fadila, B.; Samir, B. Nat. Prod. Res. 2017, 31, 2042.

[19] Tundis, R.; Deguin, B.; Loizzo, M. R.; Bonesi, M.; Statti, G. A.; Tillequin, F.; Menichini, F. Bioorg. Med. Chem. Lett. 2005, 15, 4757.

[20] Chen, Z.-W.; Hu, J.-Z. Chin. J. Org. Chem. 2006, 26, 813 (in Chinese).

(陈志卫, 胡家洲, 有机化学, 2006, 26, 813.)

[21] Quintin, J.; Lewin, G. J. Nat. Prod. 2004, 67, 1624.

[22] Faras, L.; Strelisky, J. Tetrahedron Lett. 1970, 187.

[23] Jiang, X. Y.; Li, X. C.; Liu, W. Y.; Xu, Y. H.; Feng, F.; Qu, W. Tetrahedron 2017, 73, 1895 .

[24] Yan, S. Q.; Ding, N.; Zhang, W.; Wang, P.; Li, Y. X.; Li, M. Carbohydr. Res. 2012, 354, 6.

[25] Zhang, M.; Du, Y.; Kong, F. Carbohydr. Res. 2001, 330, 319.

[26] Kang, M. M.; Ma, Z. L.; Liu, B.; Pan, D.; Li, J. Q. Chin. J. Org. Chem. 2017, 37, 1516 (in Chinese)

(康满满，马志龙，刘彪，潘登，李建其，有机化学，2017，37, 1516.)

[27] Maharik, N. A.; Botting, N. P. Eur. J. Org Chem. 2008, 5622.

(Cheng, F.) 